\title{
PROSPECTIVE EVALUATION OF STAPHYLOCOCCUS AUREUS ANTIBIOTIC RESISTANCE PATTERNS FROM RESPIRATORY SPECIMENS IN ICU PATIENTS
}

Andrew C. Bernard, MD; Associate Professor; Department of Surgery; University of Kentucky College of Medicine; Lexington, KY

Sue Overman, MA, SM (ASCP); Chief Medical Tech; UK Healthcare Clinical Microbiology; Department of Pathology and Laboratory Medicine; University of Kentucky College of Medicine; Lexington, KY

P. Shane Winstead, PharmD; Assistant Professor; Department of Pharmacy Practice and Science, University of Kentucky College of Pharmacy; Lexington, KY

Anna K. Rockich, PharmD; Assistant Professor; Department of Surgery, University of Kentucky College of Medicine; Lexington, KY

Paul A. Kearney, MD; Professor; Department of Surgery, University of Kentucky College of Medicine; Lexington, KY

Craig Martin, PharmD; Associate Professor; Department of Pharmacy Practice and Science; University of Kentucky College of Pharmacy; Lexington, KY

Julie A. Ribes, MD, PhD; Associate Professor and Director of Clinical Microbiology; Associate Director of Hospital Laboratories; Department of Pathology and Laboratory Medicine; University of Kentucky College of Medicine; Lexington, KY

- $\quad$ All work was performed at the University of Kentucky.

- This work was supported by an investigator-initiated research grant from Pfizer.

Address correspondence to: Andrew C. Bernard, M.D.

Department of Surgery, Section on Acute Care Surgery University of Kentucky College of Medicine

800 Rose Street, C-234 Lexington, Kentucky 40536-0298

Office Phone: 859-323-6346, Ext 224 859Fax: 859-323-6840 acbern00@uky.edu 


\section{ABSTRACT}

Background: Staphylococcus aureus is the leading pathogen responsible for hospital-acquired pneumonia (HAP). Vancomycin is the primary antimicrobial choice for methicillin-resistant $S$. aureus (MRSA) HAP worldwide, but $S$. aureus isolates have been reported susceptible or even resistant to vancomycin.

Objectives: This study compares automated and non-automated susceptibility patterns for MRSA isolates to vancomycin to determine comparability of testing methods for this drug.

Methods: Respiratory samples submitted to Clinical Microbiology from patients in the ICU were plated onto sheep blood agar and chocolate agar media and visually inspected at 12-24 hours. The presence of $S$. aureus was determined serologically. S. aureus isolates were subcultured for susceptibility testing to detect MRSA respiratory isolates. Consecutive MRSA respiratory isolates were analyzed for susceptibility and minimum inhibitory concentration (MIC) using E-test strips and BD Phoenix automated system testing for vancomycin and the vancomycin alternative agents teicoplanin, linezolid and tigecycline.

Results: Ninety-five MRSA respiratory isolates were collected. Three were obtained by bronchoalveolar lavage and the remainder by protected alveolar lavage. There was no heteroresistance detected by E-test at 24 hours, but there were 4 isolates at 48 hours demonstrating elevated vancomycin MIC values of $6 \mathrm{ug} / \mathrm{ml}$.

Conclusions: Vancomycin heteroresistance was not a problem in this series of isolates. Agreement between vancomycin automated MICs and E-test MICs was not universal. Although under-calls were few based upon automated MIC, several instances were indeed identified. These data show that Phoenix under-reports vancomycin MIC values for MRSA compared to Etest methodology.

Keywords: Methicillin-resistant Staphylococcus aureus; antibiotic resistance; hospital-acquired pneumonia 


\section{INTRODUCTION}

Staphylococcus aureus (S. aureus) has become the leading pathogen responsible for hospital-acquired pneumonia (HAP) and is responsible for about $20 \%$ of cases (NNIS System Report, 1999). S. aureus may be either susceptible or resistant to oxacillin/methicillin resistant $S$. aureus (MRSA). Oxacillin resistance rates are increasing, and the average rate of resistance to oxacillin currently exceeds $55 \%$ on average nationwide (Jones, et al., 2004; Rosenthal, et al, 2010).

Presence or absence of risk factors for MRSA determines whether or not MRSA drug therapy is used (American Thoracic Society, 2005). If a patient is thought to have pneumonia and they possess risk factors for MRSA, then antimicrobial therapy active against MRSA will be used empirically and "de-escalated" if objective culture data does not demonstrate MRSA. Empiric or culture-based therapy for MRSA HAP includes one of the following: 1) the glycopeptide vancomycin, or 2) the oxazoladinone linezolid.

Adequate empiric antibiotic therapy improves outcome in patients with pneumonia (Alvarez-Lerma, 1996; Dupont, et al., 2001; Kolleff, et al., 1999). Vancomycin is the primary antimicrobial choice for MRSA HAP worldwide. It is relatively inexpensive in per-dose pricing, generally effective, and has a favorable safety profile. However, S. aureus isolates which are incompletely susceptible or even resistant to vancomycin have been reported (Smith, et al., 1999). These vancomycinintermediately susceptible $S$. aureus (VISA) and vancomycin-resistant $S$. aureus (VRSA) isolates are rare but increasing (Plipat, et al., 2005). Since the first report of VISA in the United States in 1999 (Smith, et al., 1999), other reports have documented the existence of $S$. aureus isolates with incomplete susceptibility to vancomycin, termed as vancomycin "heteroresistance" (Plipat, et al., 2005).
Heteroresistant VISA (hVISA) isolates are those in which the prevalent colonies are vancomycin susceptible but in which a subpopulation of organisms demonstrate phenotypes which are intermediate or resistant to vancomycin [Plipat, et al., 2005]. HVISA has been proposed as an etiology for vancomycin treatment failure in some patients. These hVISA populations may exhibit vancomycin-susceptibility when analyzed by automated testing because automated systems are incapable of identifying rare hVISA colony forming units (CFUs) within single large inoculums (Tenover, et al., 2004). Without more detailed testing, the true incidence of hVISA isolates is unknown. No large direct comparison of automated and non-automated MRSA isolate susceptibilities to vancomycin has been reported. This investigation sought to determine the prevalence of pulmonary MRSA that are resistant or heteroresistant to vancomycin, and the prevalence of MRSA resistance to other frequently used antibiotics (i.e., linezolid, tigecycline, and teicoplanin), in a major tertiary referral center ICU setting. Vancomycin susceptibility testing results for the Phoenix automated panel (Phoenix, BD Biosciences) and the E-test strip method (BioMerieux) were directly compared; alternative agents to vancomycin (i.e., teicoplanin, tigecycline, and linezolid) were also assessed.

\section{MATERIALS AND METHODS}

This investigation was reviewed and approved by the institution's Medical IRB. Respiratory samples were collected between January 2, 2009 and April 27, 2010 by bronchoalveolar lavage (BAL) or protected alveolar lavage (PAL, CombiCath, KOL Bio Medical Instruments, Chantilly, Virginia). Samples were submitted to the Clinical Microbiology Laboratory and were cultured on sheep blood agar plates and chocolate agar plates incubated in a $\mathrm{CO}_{2}$ incubator at 
$35^{\circ} \mathrm{C}$. These plates were visually inspected at 12-24 hours. The presence of $S$. aureus was determined and $S$. aureus isolates were subcultured to fresh plates for susceptibility testing. Consecutive MRSA isolates $(n=100)$ were analyzed using E-test strips (bioMerieux, Macry L'Etoile, France) and automated microbroth dilution testing (BD Phoenix Automated Microbiology System, Franklin Lakes, NJ). S. aureus isolates that were oxacillin susceptible were excluded from further analysis.

For standard E-test susceptibility testing, isolates were diluted in broth to 0.5 McFarland turbidity, and $0.2 \mathrm{ml}$ was spread onto a $90 \mathrm{~mm}$ Brain Heart Infusion (BHI) agar plate. The plates were dried briefly prior to applying the E-test strips. Plates were incubated at $35^{\circ} \mathrm{C}$, read at 24 hours, and confirmed at 48 hours. Since evidence suggests that sensitivity and specificity for the detection of hVISA are highest if the isolates are fresh and if they are set at 2.0 McFarland turbidity (Walsh, et al., 2001; Liu \& Chambers, 2003), this was the method that was used. Linezolid and teicoplanin E-test strips were obtained from Pfizer (New York, NY), and tigecycline Etest strips were obtained from Wyeth (Madison, NJ).

E-test MICs were determined by visual inspection by laboratory microbiologists according to laboratory policy and procedure. An immediate reporting mechanism was established to respond to any evidence of antimicrobial heteroresistance so that alteration in therapy could be made as clinically indicated; no visible heteroresistance was reported during the study period. Microbiologists reading Etest results carefully examined plates for evidence of hazes, feathery growth, or heteroresistant microcolonies, according to the manufacturer's instructions. The interpretation of the test is as follows: If the MIC values for vancomycin and teicoplanin were $<6 \mathrm{mcg} / \mathrm{ml}$, the Staphylococcus was considered susceptible to vancomycin. MIC values $\geq 32 \mathrm{mcg} / \mathrm{ml}$ were considered resistant for vancomycin and teicoplanin. An isolate was considered positive for heteroresistance if vancomycin and teicoplanin MIC values were $\geq 8 \mathrm{mcg} / \mathrm{ml}$, or if the teicoplanin MIC value alone was $\geq 12$ $\mathrm{mcg} / \mathrm{ml}$. Positive results based on a teicoplanin result of 12 or $16 \mathrm{mcg} / \mathrm{ml}$ were confirmed by repeating the test. Susceptible break points of $\leq 4.0 \mathrm{mcg} / \mathrm{mL}$ and $\leq 0.5$ $\mathrm{mcg} / \mathrm{mL}$ were used for linezolid and tigecycline, respectively.

\section{RESULTS}

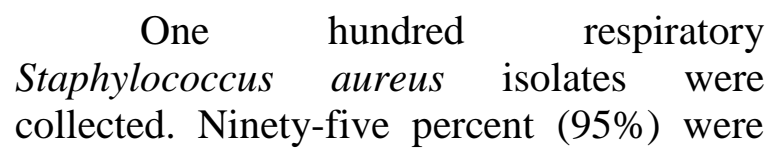
MRSA, indicating a 5\% rate of protocol deviation in the microbiology lab. Three were obtained by BAL and the others (92/95, $96.8 \%)$ by PAL. MRSA MIC values for vancomycin are shown in Table 1. Using the standard E-test methodology (testing at $0.5 \mathrm{McF}$ arland turbidity), there were no vancomycin MIC values greater than 2 $\mathrm{mcg} / \mathrm{ml}$. Using 24-hour incubation and heteroresistance methodology with 2.0 McFarland turbidity, all isolates demonstrated MIC values of $\geq 2 \mathrm{mcg} / \mathrm{ml}$ with 7/92 (7.6\%) demonstrating MIC values of $6 \mathrm{mcg} / \mathrm{ml}$. At 48 hours, heteroresistance testing demonstrated MIC values of 6 $\mathrm{mcg} / \mathrm{mL}$ for $3 / 95$ isolates $(3.2 \%)$. No E-test results were $\geq 8 \mathrm{mcg} / \mathrm{ml}$ by any method. Vancomycin MIC results by Phoenix ranged from $0.5 \mathrm{mcg} / \mathrm{ml}$ to $4 \mathrm{mcg} / \mathrm{ml} ; 98.9 \%$ of MRSA isolates had MIC values of $\leq 2$ $\mathrm{mcg} / \mathrm{ml}(91 / 92$ isolates tested) 


\begin{tabular}{|c|c|c|c|c|}
\hline \multicolumn{5}{|c|}{ Table 1. MRSA MICs for Vancomycin } \\
\hline $\begin{array}{c}\text { MIC } \\
\text { Result } \\
(\mathbf{m c g} / \mathbf{m l})\end{array}$ & $\begin{array}{c}\text { Standard } \\
\text { Methodology } \\
\text { E-test } \\
\text { n=95 }\end{array}$ & $\begin{array}{c}\text { Heteroresistance } \\
\text { Methodology E-test } \\
\text { at 24 Hours } \\
\text { n=92 }\end{array}$ & $\begin{array}{c}\text { Heteroresistance } \\
\text { Methodology E-test } \\
\text { at 48 Hours } \\
\text { n=95 }\end{array}$ & $\begin{array}{c}\text { Phoenix } \\
\text { Microbroth } \\
\text { Dilution } \\
\text { n=92 }\end{array}$ \\
\hline $\mathbf{0 . 5}$ & - & - & - & $7 / 92(7.6 \%)$ \\
\hline $\mathbf{1}$ & $12 / 95(13 \%)$ & - & - & $63 / 92(68.5 \%)$ \\
\hline $\mathbf{1 . 5}$ & $47 / 95(49 \%)$ & - & $11 / 95(11.6 \%)$ & $21 / 92(22.8 \%)$ \\
\hline $\mathbf{2}$ & $36 / 95(38 \%)$ & $23 / 92(25 \%)$ & $54 / 95(56.8 \%)$ & - \\
\hline $\mathbf{3}$ & - & $62 / 92(67.4 \%)$ & $27 / 95(28.4 \%)$ & $1 / 92(1.1 \%)$ \\
\hline $\mathbf{4}$ & - & $7 / 92(7.6 \%)$ & $3 / 95(3.2 \%)$ & - \\
\hline $\mathbf{6}$ & - & - & & - \\
\hline
\end{tabular}

Teicoplanin MIC results using E-test are shown in Table 2. As with vancomycin, results varied by method used with heteroresistance methodology and longer incubation showing higher
MIC values overall. No teicoplanin heteroresistance (MIC values $\geq 12 \mathrm{mcg} / \mathrm{ml}$ ) was seen.

\begin{tabular}{|c|c|c|c|}
\hline \multicolumn{4}{|c|}{ Table 2. MRSA MICs for Teicoplanin } \\
\hline $\begin{array}{c}\text { MIC } \\
\text { Result } \\
(\mathrm{mcg} / \mathrm{ml})\end{array}$ & $\begin{array}{c}\text { Standard } \\
\text { Methodology } \\
\text { E-test } \\
\text { n=95 }\end{array}$ & $\begin{array}{c}\text { Heteroresistance } \\
\text { Methodology } \\
\text { E-test at } 24 \text { Hours } \\
\text { n=92 }\end{array}$ & $\begin{array}{c}\text { Heteroresistance } \\
\text { Methodology } \\
\text { E-test at } 48 \text { Hours } \\
n=95\end{array}$ \\
\hline$\leq 0.5$ & $44 / 95(46.3 \%)$ & $2 / 92(2.2 \%)$ & $2(2.1 \%)$ \\
\hline 0.75 & $15 / 95(15.8 \%)$ & - & - \\
\hline 1 & $10 / 95(10.5 \%)$ & $1 / 92(1.1 \%)$ & - \\
\hline 1.5 & $15 / 95(15.8 \%)$ & $2 / 92(2.2 \%)$ & $2 / 95(2.1 \%)$ \\
\hline 2 & $9 / 95(9.5 \%)$ & $11 / 92(12.0 \%)$ & $3 / 95(3.2 \%)$ \\
\hline 3 & $1 / 95(1.1 \%)$ & $33 / 92(35.9 \%)$ & $25 / 95(26.3 \%)$ \\
\hline 4 & - & $31 / 92(33.7 \%)$ & $33 / 95(34.7 \%)$ \\
\hline 6 & $1 / 95(\%)$ & $10 / 92(10.9 \%)$ & $20 / 95(21.1 \%)$ \\
\hline 8 & - & $2 / 92(2.2 \%)$ & $10 / 95(10.5 \%)$ \\
\hline
\end{tabular}


Tigecycline E-test MIC values are shown in Table 3, while those for linezolid are shown in Table 4. At the time of the study, neither tigecycline nor linezolid were on the Phoenix susceptibility panel so these results are not available for comparison.

\begin{tabular}{|c|c|}
\hline \multicolumn{2}{|c|}{$\begin{array}{c}\text { Table 3. MRSA MICs for Tigecycline } \\
\text { Standard Methodology E-test (n=95) }\end{array}$} \\
\hline MIC Result (mcg/ml) & Number of Isolates (\%) \\
\hline $\mathbf{0 . 0}$ & $1 / 92(1.1 \%)$ \\
\hline $\mathbf{0 . 0 6}$ & $3 / 9(3.2 \%)$ \\
\hline $\mathbf{0 . 0 9}$ & $19 / 95(20 \%)$ \\
\hline $\mathbf{0 . 1 2 5}$ & $12 / 95(12.6 \%)$ \\
\hline $\mathbf{0 . 1 3}$ & $7 / 95(7.4 \%)$ \\
\hline $\mathbf{0 . 1 9}$ & $18 / 95(18.9 \%)$ \\
\hline $\mathbf{0 . 2 5}$ & $15 / 95(15.8 \%)$ \\
\hline $\mathbf{0 . 2 8}$ & $1 / 95(1.1 \%)$ \\
\hline $\mathbf{0 . 3 8}$ & $7 / 95(7.4 \%)$ \\
\hline $\mathbf{1}$ & $8 / 95(8.4 \%)$ \\
\hline $\mathbf{1 . 5}$ & $2 / 95(2.1 \%)$ \\
\hline $\mathbf{2}$ & $2 / 95(2.1 \%)$ \\
\hline
\end{tabular}

\begin{tabular}{|c|c|}
\hline \multicolumn{2}{|c|}{$\begin{array}{c}\text { Table 4. MRSA MICs for Linezolid } \\
\text { Standard Methodology E-test (n=95) }\end{array}$} \\
\hline MIC Result (mcg/ml) & Number of Isolates (\%) \\
\hline $\mathbf{0 . 2 5}$ & $1 / 95(1.1 \%)$ \\
\hline $\mathbf{0 . 3 8}$ & $1 / 95(1.1 \%)$ \\
\hline $\mathbf{0 . 5}$ & $6 / 95(6.3 \%)$ \\
\hline $\mathbf{0 . 7 5}$ & $21 / 95(22.1 \%)$ \\
\hline $\mathbf{1}$ & $30 / 95(31.6 \%)$ \\
\hline $\mathbf{1 . 5}$ & $9 / 95(9.5 \%)$ \\
\hline $\mathbf{2}$ & $21 / 95(22.1 \%)$ \\
\hline $\mathbf{3}$ & $5 / 95(5.3 \%)$ \\
\hline $\mathbf{4}$ & $1 / 95(1.1 \%)$ \\
\hline
\end{tabular}




\section{DISCUSSION}

While the prevalence of MRSA among all $S$. aureus isolates is increasing, in vitro evidence of growing resistance of MRSA isolates to vancomycin is also increasing. In vitro susceptibility testing for any organism and antimicrobial is expressed as minimal inhibitory concentration (MIC). Vancomycin MICs for MRSA are rising (Wang, et al., 2003). S. aureus isolates for which vancomycin MICs are $4-8 \mu \mathrm{g} / \mathrm{mL}$ are classified as vancomycin-intermediate, and isolates for which vancomycin MICs are $\geq 16 \mu \mathrm{g} / \mathrm{mL}$ are classified as vancomycinresistant by the Centers for Disease Control (Centers for Disease Control and Prevention).

Automated equipment historically does not report specific vancomycin MIC values below or equal to $2 \mathrm{ug} / \mathrm{ml}$. The limitation with such equipment is that as vancomycin MIC values for MRSA have crept upward over time, we now have automated equipment that reports all MRSA isolates with vancomycin MIC values $\leq 2$ $\mu \mathrm{g} / \mathrm{mL}$ as susceptible, with no distinction of whether the MIC is $0.5,1$ or $2 \mathrm{ug} / \mathrm{ml}$. Automated equipment generally reports MIC values in doubling dilutions. Furthermore, while the majority of the sample may contain individual cells with vancomycin MIC values in the susceptible range, we now know that susceptibility may vary from one bacterial cell to another within a given sample. Some solitary organisms or CFUs may demonstrate a phenotype in which vancomycin MIC values are higher than the majority of the sample, but their numbers are so small as to be unrecognized in the microbroth dilution assay. This variability in MRSA resistance to vancomycin is termed heteroresistance, and heteroresistance may contribute to treatment failure. Moreover, ideal methods to detect and address vancomycin heteroresistance in $S$. aureus in every institution or laboratory have yet to be established. Data shown here indicate that methodology is clearly critical when it comes to E-testing. The organism concentration, drug used for testing, and length of incubations all play a role in the detection of heteroresistance within a bacterial population.

These data show that Phoenix underreports vancomycin MIC values for MRSA compared to E-test methodology. Thirtyeight percent of the E-test results showed a vancomycin MIC of $2 \mathrm{mcg} / \mathrm{ml}$ while only $23.9 \%$ of automated isolates demonstrated vancomycin MIC of $2 \mathrm{mcg} / \mathrm{ml}$. To determine the prevalence of MRSA isolates with marginal susceptibility to vancomycin or heteroresistance was the primary aim of this investigation. None was found. While there were no misses of heteroresistance by Phoenix testing, none were detected by either method employed in this study. These data are limited in that the isolates are derived from a single institution and must therefore be considered local. Admittedly, the sample size of 95 isolates may have been inadequate to detect the rare heteroresistant isolate found at this institution, particularly in our institution where oxacillin resistance rates in S. aureus isolates is lower than the National Nosocomial Infections Surveillance (NNIS)-reported national average of $56 \%$ (NNIS, 1999).

Since adequate empiric therapy determines outcome, it is imperative that clinicians treat patients at risk for MRSA with antimicrobials certain to eradicate MRSA. Traditional therapy was almost exclusively the glycopeptide vancomycin, but emerging evidence suggests that in some cases vancomycin is not as effective as other available drugs. Kollef et al. reported improved clinical cure and survival in patients with MRSA ventilator-associated pneumonia (VAP) who were treated with linezolid compared to those treated with vancomycin (survival odds ratio 4.6 for 
linezolid vs vancomycin) (Kollef, et al., 2004)]. Improved microbiologic eradication and reduced mortality has also been shown in MRSA bacteremia treated with linezolid compared to vancomycin (12\% mortality with linezolid vs $40 \%$ mortality for vancomycin) (Gomez, et al., 2007).

We found that all isolates analyzed had MICs for tigecycline and linezolid that were in the therapeutic range. Chung et al. reported the same in a series of hVISA isolates (Chung, et al., 2007), and Hortiwakul et al. also reported $100 \%$ susceptibility to linezolid in 100 MRSA isolates from patients (Hortiwakul, et al., 2012).

Teicoplanin resistance did not occur in our series. Wilson et al. reported reduced susceptibility to teicoplanin, defined by MIC $>16$, in $3.3 \%$ of 643 MRSA strains (Wilson, et al., 2006). Our series was smaller but affirms the findings that teicoplanin resistance among MRSA strains is relatively low.

Isolates in this study were only cultured for a limited period of time, and it is possible that further culture may have revealed evolving resistance pattern. Webster et al. reported a MRSA isolate with an MIC changing from $\leq 1$ to $4 \mathrm{ug} / \mathrm{ml}$ over several months, demonstrating the ability of MRSA to acquire or alter its resistance patterns over times (Webster, et al., 2007). 


\section{REFERENCES}

Alvarez-Lerma F. Modification of empiric antibiotic treatment in patients with pneumonia acquired in the intensive care unit. ICU-Acquired Pneumonia Study Group. Intensive Care Med. 1996;22(5):387-394.

http://www.ncbi.nlm.nih.gov/pubmed/?term $=$ Alvarez-

Lerma+F\% 2C+Modification+of+empiric+a ntibiotic+treatment

American Thoracic Society and Infectious Disease Society of America. Guidelines for the management of adults with hospitalacquired, ventilator-associated, and healthcare-associated pneumonia. $A m J$ Respir Crit Care Med. 2005;171(4):388416.

http://www.atsjournals.org/doi/abs/10.1164/ rccm.200405-644ST?url_ver=Z39.882003\&rfr_id=ori:rid:crossref.org\&rfr_dat $=c$ r_pub\%3dpubmed\#.VPzNOU3wvIU

Centers for Disease Control and Prevention, CDC Home. Laboratory Detection of Vancomycin-Intermediate/Resistant Staphylococcus aureus (VISA/VRSA), last revised August 6, 2012. Available at: http://www.cdc.gov/HAI/settings/lab/visa_v rsa_lab_detection.html. Accessed August 24, 2012.

Chung G, Cha J, Han S, et al. Nationwide surveillance study of vancomycin intermediate Staphylocuc aureus strains in Korean hospitals from 2001 to 2006. $J$ Microbiol Biotechnol. 2006;20(3):637-642. http://www.jmb.or.kr/journal/viewJournal.ht $\underline{\text { ml} \text { ? year }=2010 \& \mathrm{vol}=20 \& \text { num }=3 \& \text { page }=637}$

Dupont H, Mentec H, Sollett JP, Bleichner G. Impact of appropriateness of initial antibiotic therapy on the outcome of ventilator-associated pneumonia. Intensive Care Med. 2001;27(2):355-362. http://www.ncbi.nlm.nih.gov/pubmed/?term
$=$ Dupont $+\mathrm{H} \% 2 \mathrm{C}+$ Mentec $+\mathrm{H} \% 2 \mathrm{C}+$ Sollett $\mathrm{J}$ $\underline{\mathrm{P}}$

Gomez J, Garcia-Vazquez E, Banos R, et al. Predictors of mortality in patients with methicillin-resistant Staphylococcus aureus (MRSA) bacteraemia: the role of empiric antibiotic therapy. Eur J Clin Microbiol Infect Dis. 2007;26(4):239-245. http://www.ncbi.nlm.nih.gov/pubmed/17318 $\underline{479}$

Hortiwakul T, Nagij S, Chusri S, Slipaprojakul K. Nosocomial bloodstream infection in Songklandagarind; outcome and factors influencing prognosis. $J$ Med Assoc Thai. 2012;95(2):170-174. http://www.ncbi.nlm.nih.gov/pubmed/?term= Hortiwakul+T\%2C+Nagij+S\%2C+Chusri+S

Jones ME, Draghi DC, Thornsberry C, Karlowsky JA, Sahn DF, Wenzel RP. Emerging resistance among bacterial pathogens in the intensive care unit - a European and North American

Surveillance study (2000-2002). Ann Clin Microbiol Antimicrob. 2004;3:14. http://www.ncbi.nlm.nih.gov/pmc/articles/P $\underline{\text { MC509280/ }}$

Kollef MH, Sherman G, Ward S, Fraser VJ. Inadequate antimicrobial treatment of infections: a risk factor for hospital mortality among critically ill patients. Chest. 1999;115:462-474. http://www.ncbi.nlm.nih.gov/pubmed/11057 $\underline{801}$

Kollef MH, Rello J, Cammarata SK, CroosDabrera RV, Wunderink RG. Clinical cure and survival in Gram-positive ventilatorassociated pneumonia: retrospective analysis of two double-blind studies comparing linezolid with vancomycin. Intensive Care Med. 2004;30(3):388-394. http://www.ncbi.nlm.nih.gov/pubmed/14714 $\underline{107}$ 
Liu C, Chambers HF. Staphylococcus aureus with heterogeneous resistance to vancomycin: epidemiology, clinical significance, and critical assessment of diagnostic methods. Antimicrob Agents Chemother. 2003;47:30403045.

http://www.ncbi.nlm.nih.gov/pmc/articles/PM $\underline{\mathrm{C} 201119 /}$

National Nosocomial Infections Surveillance (NNIS) System report, data summary from January 1990-May 1999, issued June 1999. Am J Infect Control. 1999;27(6):520-532.

http://www.sciencedirect.com/science/articl e/pii/S0196655399700313

Plipat N, Livni G, Bertram H, Thomson RB Jr. Unstable vancomycin heteroresistance is common in clinical isolates of methicillinresistant staphylococcus aureus. J Clin Microbiol. 2005;43(5):2494-2496. http://www.ncbi.nlm.nih.gov/pubmed/?term =Plipat+N\%2C+Livni+G\%2C+Bertram+H

Rosenthal VD, Maki DG, Jamulitrat S, et al; INICC Members. International Nosocomial Infection Control Consortium (NICC) report, data summary for 2003-2008, issued June 2009. Am J Infect Control. 2010;38(2):95-104.e.2.

http://www.sciencedirect.com/science/articl e/pii/S0196655309009523

Smith TL, Pearson ML, Wilcox KR, et al. Emergence of vancomycin resistance in Staphylococcus aureus. GlycopeptideIntermediate Staphylococcus aureus Working Group. $N$ Eng $J$ Med.1999;340(7):493-501.

http://www.nejm.org/doi/full/10.1056/NEJ $\underline{\text { M199902183400701 }}$

Tenover FC, Weigel LM, Appelbaum PC, et al. Vancomycin-resistant Staphylococcus aureus isolate from a patient in Pennsylvania. Antimicrob Agents Chemother. 2004;48(1):275-280.
http://www.ncbi.nlm.nih.gov/pmc/articles/P MC310200/

Walsh TR, Bolmstrom A, Qwarnstrom A, et al. Evaluation of current methods for detection of Staphylococci with reduced susceptibility to glycopeptides. $J$ Clin Microb. 2001;39(7):2439-2444.

Wang G, Hindler JF, Ward KW, Bruckner DA. Increased vancomycin MICs for Staphylococcus aureus clinical isolates from a university hospital during a 5-year period. J Clin Microbiol. 2006;44(11):3883-3886. http://www.ncbi.nlm.nih.gov/pmc/articles/P MC1698298/

Webster D, Rennie RP, Brosnikoff CL, Chui L, Brown C. Methicillin-resistant Staphylococcus aureus with reduced susceptibility to vancomycin in Canada. Diagn Microbiol Infect Dis. 2007; 57(2):177-181.

http://www.sciencedirect.com/science/articl e/pii/S0732889306002549

Wilson AP, Cepeda JA, Hayman S, Whitehouse T, Singer M, Bellingan G. In vitro susceptibility of Gram-positive pathogens to linezolid and teicoplanin and effect on outcomes in critically ill patients. $J$ Antimicrob Chemother, 2006;58(2):470473.

http://jac.oxfordjournals.org/content/58/2/47 $\underline{0 . \text { long }}$ 